\title{
Thermal \& Mechanical Analysis of Silver Nanoparticle/Graphene Nanocomposite
}

\author{
Arvind Kumar
}

\begin{abstract}
These days the application and use of the composite products are rising exponentially in the various engineering fields due to their balanced characteristics because of the great strength to the weight ratio are the crucial characteristics. The purpose of this study/research is to develop an economical compound/composite material with improved mechanical \& thermal characteristics. Styrene Acrylonitrile $(A S N)$ is an economical, transparent (see through), rigid, chemically \& thermally steady material. The stable material propose the solution with the assistance of Silver Nanoparticles (Ag-NP) \& Graphene. The projected nanoparticle is ready by "One Step Melt Compounding Method" of SAN, Ag-NP \& Graphene in co-rotating twin screw extruder in various lots with various weight fractions (wt. \%) of the Ag-NP \& graphene subsequently, and the specimen preparation was done by the compression modeling. Tensile test is executed to execute mechanical properties while Thermogravimetric study/Analysis (TGA) is done to consider/evaluate the thermal degradation temperature range. Assess of morphology of surface of the nanoparticle/nanocomposites is executed by the Scanning Electron Microscopy (SEM). These properties of material provides basis to design the product with greater mechanical \& thermal characteristics.
\end{abstract}

Keywords: Styrene Acrylonitrile (SAN), Graphene, Silver nanoparticles (AgNP), Thermo-gravimetric Analysis (TGA), Scanning Electron Microscopy (SEM)

\section{INTRODUCTION}

The properties \& applications of polymer systems by modifying or copolymerizing with other polymers can be adjusted to a great extent to allow the performance of the resulting materials to the first standards Thus, in recent ages, an interesting shot has been made to exploit the potential use of various copolymers for mixing \& sometimes even the potential useful properties of each component to yield invalid properties. The characteristics \& applications of polymer systems by modifying or comparing them with other polymers can be varied, to a great extent, so that the performance of the resulting materials can meet leading standards. Therefore, an interesting attempt is being focused towards the interest of the various complexes of mixing and sometimes the potential for the useful properties of each component to be reached at the level of invalid properties [1]-[4].

Matter is the source of human evolution and together with humans, materials also evolved. Nowadays polymer is the most versatile/resourceful material as the properties \& applications of polymer materials can be reformed to a highly wasteful range as it can be effortlessly modified to encounter the desired standards. One of the ways to change the polymer properties is by adding a strengthening material.

Revised Manuscript Received on September 14, 2019.

Arvind Kumar, Research: Department of Mechanical Engineering, Sanskriti University, UttarPradesh India. (E-mail: sanpubip@gmail.com)
These strengthening materials are typically blend/inserted to matrix to boost the mechanical \& thermal properties [5] [10].

But now through the introduction of nanoparticles, this property boom has attained new heights. These constituents have a particularly extraordinary surface area by reason of their small sizes and thus the interaction with the matrix is better than ever \& therefore converts characteristics better than other conventional strengthening materials. Graphene, unique sexiest atoms of carbon held in hexagonal bonds, is a high-strength nonconductive material with superior thermal $\&$ electrical conditioning. Attributable to its exclusive physical, \& chemical \& biological characteristics, silver nanoparticles are widely employed in numerous fields such as medical, \& food, \& health \& industrial [11]-[15].

\section{MATERIALS \& METHOD OF THE EXPERIMENT}

\section{Materials Used:}

The proposed Nano-complex consists of:

$>\quad$ Sryrene Acrylonitrile (SAN) provided by INEOS Styrolution India Ltd., Vadodara, \& Gujarat.

$>\quad$ Silver Nanoparticles (AgNP) Available from Nano Research/study Lab., Jamshedpur, \& Jharkhand.

$>$ Graphene is available from Research Nano, Jamshedpur, \& Jharkhand.

\section{Method Operated for the Experiment:}

The proposed nanocomposite design begins with the preparation of different coatings by applying different matrices \& reinforcing materials in diverse weight scales $(\%)$.

\begin{tabular}{|c|c|c|c|}
\hline & SAN & Graphene & AgNP \\
\hline Batch 1 & 100 & 0 & 0 \\
\hline Batch2 & 98 & 1 & 1 \\
\hline Batch 3 & 96 & 2 & 2 \\
\hline
\end{tabular}

Figure 1. Batch Composition in wt. \%

The proposed nanocomposite is fabricated by an assortment of layers of SAN, AgNP \& graphene. The SAN / Ag-NP / Graphene is chained into a foil chassis and then transferred to a subsequent extruded chip containing its devices, whose settings are as underneath:

Published By:

Blue Eyes Intelligence Engineering

\& Sciences Publication 


\begin{tabular}{|l|l|l|l|l|l|}
\hline & Zone 1 & Zone 2 & Zone 3 & Zone 4 & Zone 5 \\
\hline $\begin{array}{l}\text { Temp. } \\
(\mathrm{C})\end{array}$ & 210 & 220 & 230 & 250 & 260 \\
\hline
\end{tabular}

Extruder speed : 40 RPM

Figure 2. Extruder Temperature

The extrudate that came out of a screw couple die extruder is in the form of a wire that is pulled out and cooled to room temperature in a water bath $\&$ then transformed into an essential size pallet using a palletizer. Then, for sampling, these pallets were prepared at $80^{\circ} \mathrm{C}$ for 2 hours in the heating oven and burned to burn the contents of the mantle, and then these pellets were anchored in the workplace by a compressor sealer at their temperature. $230^{\circ} \mathrm{C}$ and a pressure of $100 \mathrm{ps}$ are washed for 4 minutes before cooling. The compression molding exit is a box of $180 \times 150 \mathrm{~cm}$ file that is used on the sample size required and returns using the contour.

\section{TESTING}

\section{Tensile Test}

All samples are tensile tested using Universal Testing Machine (UTM) according to the ASTM D638 standard to evaluate tensile strength of the nanocomposite i.e. to determine how much uniaxial pressure it can withstand before failure.

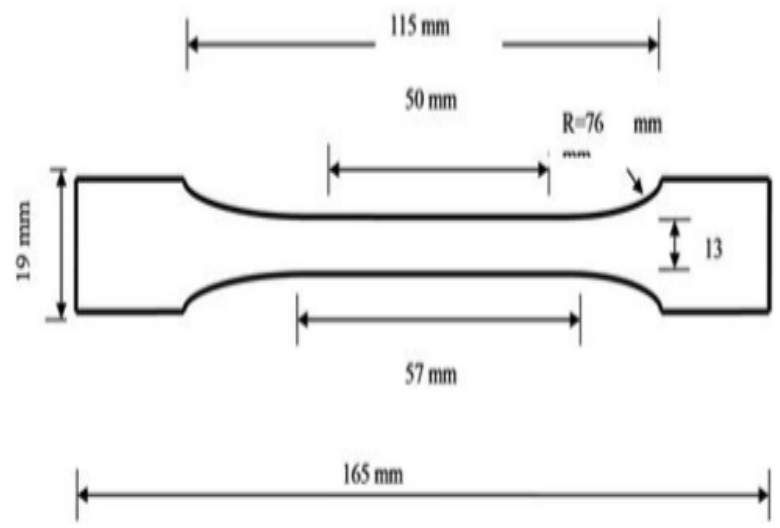

Figure 3. Tensile Test Specimen

Test speed $-50.0 \mathrm{~mm} / \mathrm{min}$

Thermo-gravimetric Study/Analysis:

TGA is a heat analyzer that can be used for mass analysis or sample weight as a purpose of temperature (in scanning mode) \& as a purpose of time (in). It is a technology that is used to basically find the thermal solidity/stability of the material.

Test conditions:

Minimum temperature: $0^{0} \mathrm{C}$

Minimum temperature: $800^{\circ} \mathrm{C}$

Rate: $20^{\circ} \mathrm{C} / \mathrm{min}$.

\section{Scanning Electron Microscopy}

SEM is the kind of instrument that produces image of the sample/section through scanning with the concentrated beam of electron. SEM is done to analyse the surface/exterior morphology of specimen. For this test the fractured/distorted surface from the tensile test is used for scanning.

\section{RESULTS \& DISCUSSION}

Tensile Test

\begin{tabular}{|c|c|c|}
\hline & $\begin{array}{c}\text { Density } \\
\left(\mathrm{g} / \mathrm{cm}^{3}\right)\end{array}$ & Tensile Strength $(\mathrm{MPa})$ \\
\hline Batch 1 & 1.04 & 68.2 \\
\hline Batch 2 & 1.1468 & 82.3 \\
\hline Batch 3 & 1.253 & 54.8 \\
\hline
\end{tabular}

Figure 4. Tensile Strength

A good mechanical strength is an outstanding feature of what kind of material can be employed to make any product available. It is apparent in this research/study that the nanomaterials were compatible with the SAN core materials at low concentrations \& thus produced strong mechanical strength but as the Graphene and AgNP loading percentage exceeded $2 \% \mathrm{wt}$. could be the starting properties as the material sounds, basically are completely mature and thus excessive Nano material may cause the material to collapse quickly.

\section{Thermo-gravimetric Study/Analysis}

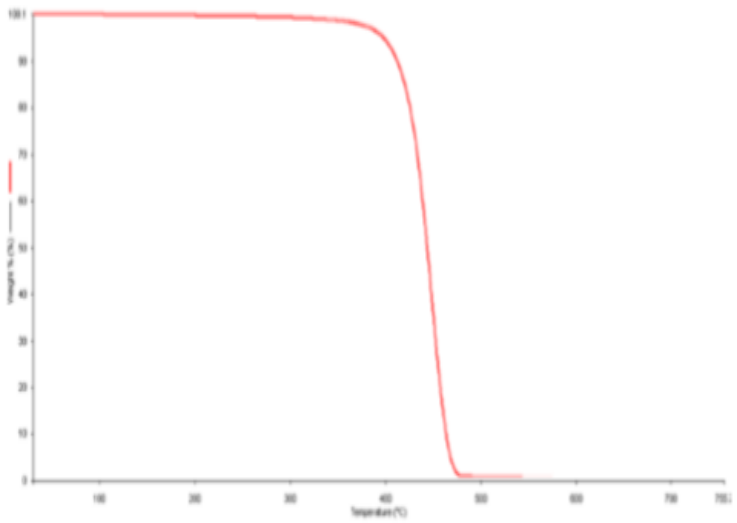

Figure 5. TAG of SAN

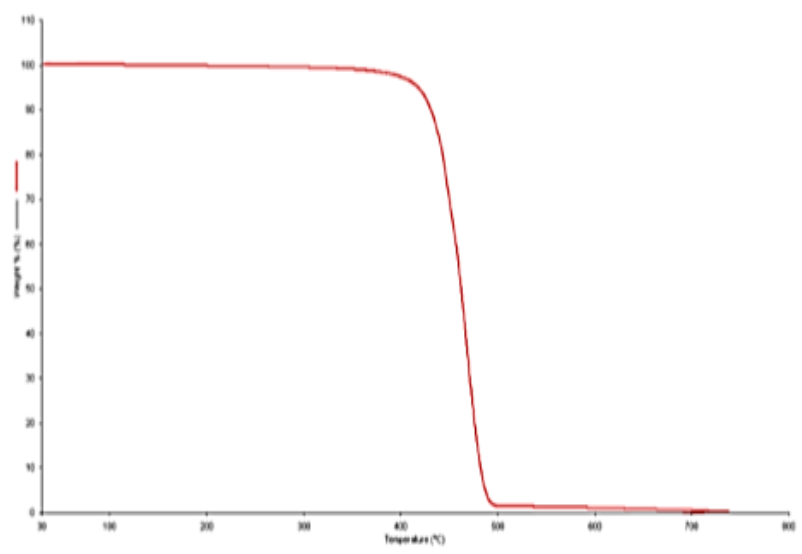

Figure 6. TGA of SAN+ 1\% Graphene+ 1\% Ag-NP

Published By: 


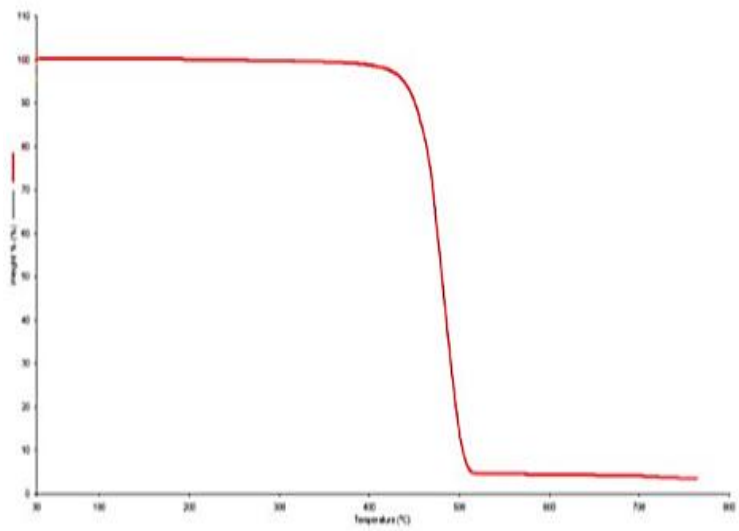

Figure 7. TGA+2\% Graphene+2\% Ag-NP

TGA analysis/study of the specimen/samples reveals that with increasing loading Graphene and Ag-NP increase the thermal stability/solidity of the nanocomposite. This is as a result of the reality that Graphene \& Ag-NP have very high thermal stability \& may lead to an increase in the chemical stability/solidity of the coating. The SAN degradation rate started from $400^{\circ} \mathrm{C}$ to around $480^{\circ} \mathrm{C}$ but as Graphene and $\mathrm{AgNP}$ increased, the degradation limit increased to around $510^{\circ} \mathrm{C}$

\section{Scanning Electron Microscopy}

SEM study/analysis of the sample containing SAN with 5 wt. \% of Graphene and 5wt\% of AgNP is shown in Figure $\mathbf{8}$, form this fig. only matrix that is SAN \& not the Graphene \& Ag-NP due to the size of the Graphene \& AgNP it is in the range of 1-5 nanometer which is outside the resolution power of the SEM microscope technique are used (JCM 6000 Plus). But one thing author can find from the surface author examined is a broken fracture surface.

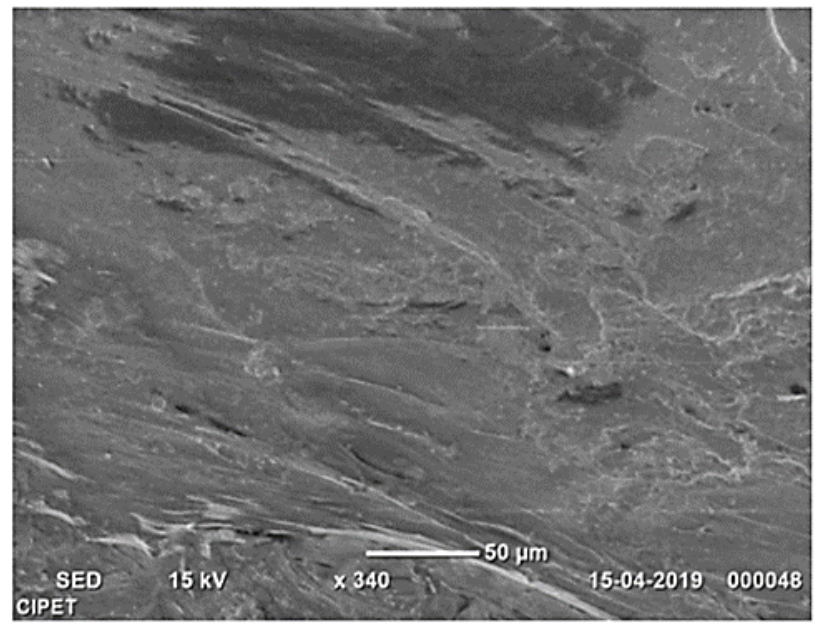

Figure 8. SEM figure/image of $\mathrm{SAN}+2 \%$ Graphene+2\% AgNP

\section{CONCLUSION}

The mechanical \& thermal experiments \& the SEM categorization of the anticipated Nano-composite revealed that the mechanical \& thermal stability of the material is greatly increased due to the synergetic influence of the Graphene \& AgNP when blended in SAN matrix. As a result of its light weight, raised strength \& better thermal properties \& other important properties it can provide figure/image is that the surface is very smooth because the

antibacterial properties \& EMI shielding due to AgNP \& Graphene \& thus this Nano-composite material/substance can be employed in electronics \& healthcare industry.

\section{REFERENCE}

1. W. Hu et al., "Graphene-Based Antibacterial Paper," 2010.

2. C. ; Huang, X. ; Qi, X. Y. Boey, and F. Y. C. Zhang, "Graphene-Based Composites," Chem. Soc. Rev., vol. 41, pp. 666-686, 2012.

3. P. Kumar, P. Huo, R. Zhang, and B. Liu, "Antibacterial Properties of Graphene-Based Nanomaterials," Nanomaterials, vol. 9, no. 5, p. 737, May 2019.

4. M. Moritz and M. Geszke-Moritz, "The newest achievements in synthesis, immobilization and practical applications of antibacterial nanoparticles," Chem. Eng J., vol. 228, pp. 596-613, Jul. 2013.

5. 'Current Topics in Nutraceutical Research.'

6. L. Shi, J. Liu, J. Yang, L. Cai, L. Shi, and H. Qiu, "Langmuir-Blodgett assembly of transparent graphene oxide-silver microwire hybrid films with an antibacterial property," New Carbon Mater., vol. 32, no. 4, pp. 344351, Aug. 2017.

7. S. Liu, "ANXA5 Enhances Malignancy of Murine Hepatocarcinoma Hca-P Cells \&amp;lt;i\&amp;gt;via\&amp;lt;/iamp;gt; $\quad$ ERK Activation and E-cadherin Suppression," Am. J. Life Sci., vol. 7, no. 1, p. 31, 2019.

8. T. Cheng-an, Z. Hao, W. Fang, Z. Hui, Z. Xiaorong, and W. Jianfang, "Mechanical Properties of Graphene Oxide/Polyvinyl Alcohol Composite Film," Polym. Polym. Compos., vol. 25, no. 1, pp. 11-16, Jan. 2017.

9. B. Das, K. Eswar Prasad, U. Ramamurty, and C. N. R. Rao, "Nano-indentation studies on polymer matrix composites reinforced by few-layer graphene,' Nanotechnology, vol. 20, no. 12, p. 125705, Mar. 2009.

10. U. Khan, P. May, H. Porwal, K. Nawaz, and J. N Coleman, "Improved Adhesive Strength and Toughness of Polyvinyl Acetate Glue on Addition of Small Quantities of Graphene," ACS Appl. Mater. Interfaces, vol. 5, no. 4, pp. 1423-1428, Feb. 2013.

11. K. Nawaz, M. Ayub, N. Ul-Haq, M. B. Khan, M. B. Khan Niazi, and A. Hussain, "The effect of large area graphene oxide (LAGO) nanosheets on the mechanical properties of polyvinyl alcohol," J. Polym. Eng., vol. 36, no. 4, pp. 399-405, Jan. 2016.

12. T. Ramanathan et al., "Functionalized graphene sheets for polymer nanocomposites," Nat. Nanotechnol., vol. 3, no. 6, pp. 327-331, Jun. 2008.

13. L. Yang et al., "A high throughput method for preparation of highly conductive functionalized graphene and conductive polymer nanocomposites," RSC $A d v$., vol. 2, no. 6, p. 2208, Feb. 2012.

14. M. Abbas, N. Naeem, H. Iftikhar, and U. Latif, "Synthesis, Characterization and Antimicrobial Properties of Silver Nanocomposites," in Silver Nanoparticles - Fabrication, Characterization and Applications, InTech, 2018.

15. M. Cobos, M. J. Fernández, and M. D. Fernández, "Graphene Based Poly(Vinyl Alcohol) Nanocomposites Prepared by In Situ Green Reduction of Graphene Oxide by Ascorbic Acid: Influence of Graphene Content and Glycerol Plasticizer on Properties.," Nanomater. (Basel, Switzerland), vol. 8, no. 12, Dec. 2018. 